BA-95-19; astro-ph/9507006

\title{
Cold Plus Hot Dark Matter Cosmology in the Light of Solar and Atmospheric Neutrino Oscillations
}

\author{
K.S. Babu色, R.K. Schaefer, and Q. Shafi \\ Bartol Research Institute \\ University of Delaware \\ Newark, Delaware 19716
}

\begin{abstract}
We explore the implications of possible neutrino oscillations, as indicated by the solar and atmospheric neutrino experiments, for the cold plus hot dark matter scenario of large scale structure formation. We find that there are essentially three distinct schemes that can accommodate the oscillation data and which also allow for dark matter neutrinos. These include (i) three nearly degenerate (in mass) neutrinos, (ii) non-degenerate masses with $\nu_{\tau}$ in the $\mathrm{eV}$ range, and (iii) nearly degenerate $\nu_{\mu}-\nu_{\tau}$ pair (in the $\mathrm{eV}$ range), with the additional possibility that the electron neutrino is cosmologically significant. The last two schemes invoke a 'sterile' neutrino which is light $(\lesssim \mathrm{eV})$. We discuss the implications of these schemes for $\bar{\nu}_{\mu}-\bar{\nu}_{e}$ and $\nu_{\mu}-\nu_{\tau}$ oscillation, and find that scheme (ii) in particular, predicts them to be in the observable range. As far as structure formation is concerned, we compare the one neutrino flavor case with a variety of other possibilities, including two and three degenerate neutrino flavors. We show, both analytically and numerically, the effects of these neutrino mass scenarios on the amplitude of cosmological density fluctuations. With a Hubble constant of $50 \mathrm{~km} \mathrm{~s}^{-1} \mathrm{Mpc}^{-1}$, a spectral index of unity, and $\Omega_{\text {baryon }}=0.05$, the two and three flavor scenarios fit the observational data marginally better than the single flavor scheme. However, taking account of the uncertainties in these parameters, we show that it is premature to pick a clear winner.
\end{abstract}

\footnotetext{
${ }^{1}$ Work supported in part by grants from the DOE and NASA.

2 Address starting September 1995: School of Natural Sciences, Institute for Advanced Study, Olden Lane, Princeton, NJ 08540
} 
PACS numbers: $\quad$ 98.65-r, 14.60-st, $95.35+\mathrm{d}$ 


\section{Introduction}

The idea that the dark matter in the universe may contain both a 'cold' as well as a 'hot' component [1] first arose as a serious possibility [2] within the framework of grand unified theories (which also inspired inflation, cosmic strings, baryogenesis, etc.). In the original models the cold component was the axion, although nowadays the lightest supersymmetric particle (LSP) often plays that role. Lightly massive neutrinos are the hot component, and it was usually assumed that the neutrinos would display the hierarchical mass spectrum characteristic of the quarks and charged leptons, with a single neutrino $\left(e . g ., \nu_{\tau}\right)$ dominating the mass density of the hot component.

However, it was noted from the beginning [1] that because of their special nature, nearly degenerate massive neutrinos is a logical, albeit not necessarily the simplest, possibility. The cosmological implications of neutrinos that are closely degenerate in mass has received much recent attention [3, [4, 5], inspired to some extent by the remarkable series of neutrino oscillation experiments (solar, atmospheric, and more recently accelerator) which suggest that two or more neutrino flavors may contribute to the hot dark matter in the universe.

Before discussing the impact of neutrino oscillation experiments on the cold plus hot dark matter $(\mathrm{C}+\mathrm{HDM})$ scenario, let us briefly recall why the latter has attracted so much recent attention. From the mid to the late 1980s evidence was mounting that the so-called "standard" cold dark matter $(\mathrm{CDM})$ scenario had trouble providing a consistent explanation of small $(\sim$ galactic) as well as large scale structure. On the contrary, a $\mathrm{C}+\mathrm{HDM}$ model 
(with $\Omega_{\nu} \sim 0.15-0.35$ ) provided a far more consistent fit to the data [6]. In order to provide additional tests, the quadrupole anisotropy of the microwave background expected in C+HDM models was estimated in 1989 [7, 8] and compared with the CDM prediction. Normalized to the 'small' scale data, it was found that the $\mathrm{C}+\mathrm{HDM}$ prediction exceeded the CDM value by a factor of about two, and this was dramatically verified when the COBE team 9] made its announcement in 1992 [10].

In the last two years, particularly after extensive numerical simulations of galaxy and cluster formation [11] and more detailed analytical work [12, 13], additional support for the $\mathrm{C}+\mathrm{HDM}$ model has emerged. It appears that this model provides the simplest (consistent) realization of an inflationary scenario for large scale structure formation. It has recently been pointed out [3], 4], (and our results are in agreement) that with two or more neutrino flavors contributing to the hot dark matter, somewhat better fits to the present data may be possible. For fixed $\Omega_{\nu}$ there is also some dependence both on the spectral index as well as the Hubble constant. It must be admitted, though, that within the framework of grand unified theories, it may not be easy to realize scenarios with two or three nearly degenerate (in mass) neutrinos. Furthermore, in some of the scenarios we consider, one needs to invoke a fourth (sterile) neutrino which is nearly degenerate in mass with the electron neutrino. Precisely why this singlet state can be so light is an important question for future research.

The layout of the paper is as follows. In section 2.1 we list three sets of observations related to solar, atmospheric, and dark matter neutrinos which 
provide hints for non-zero neutrino masses. For completeness, we also summarize the results of the LSND experiment at Los Alamos, although we will not include them among the constraints to be satisfied. We then proceed to a discussion of three distinct schemes which are consistent with the observed solar and atmospheric $\nu$ oscillations, and which also allow for neutrino dark matter. We discuss the implications of these schemes for a variety of experiments, particularly the $\bar{\nu}_{\mu}-\bar{\nu}_{e}$ and $\nu_{\mu}-\nu_{\tau}$ oscillation experiments at accelerators. In section 3 we address the main issues raised by neutrino oscillations for cosmological structure formation within the $\mathrm{C}+\mathrm{HDM}$ framework. We review the physics of the differences between having one and more than one massive neutrino flavor, giving some analytic formula to estimate the scale (section 3.1) and amplitude (section 3.2) of the effects on the growth of primordial density fluctuations. In 3.3 we give results of some more detailed numerical computations of the cosmological models based on the solutions to the oscillation data, including some models, in 3.4, motivated by the LSND results. Finally, in section 3.5 we discuss the dependence on the cosmological parameters (Hubble constant, spectral index $n$, and baryonic density fraction) of conclusions drawn from comparing specific models to large scale structure data.

\section{$2 \quad$ Neutrino Masses and Mixings}

\subsection{Observational Hints}

One of the greatest challenges in particle physics today pertains to the issue of the neutrino masses. There are indications from a variety of experiments 
that one (or more) of the known neutrinos may possess non-zero mass(es). In this section we briefly summarize the relevant observations based on solar and atmospheric neutrinos, as well as present arguments for neutrino dark matter. [For completeness, we also summarize the recent findings of the LSND experiment at Los Alamos.] In section 2.2, we discuss scenarios for neutrino masses and mixings which can simultaneously accommodate these observations. We find that there are essentially three viable schemes, and remarkably enough, all three will be tested in ongoing and planned neutrino oscillation experiments (particularly Los Alamos LSND, Rutherford KARMEN, the CERN CHORUS/NOMAD and Fermilab E803 experiments).

1. Solar neutrino puzzle: The apparent deficit in the flux of solar $\nu_{e}$ 's [14 that has persisted in the Chlorine experiment for over two decades 15] has been confirmed in the last several years by three independent experiments: the Kamiokande water Cerenkov detector [16], and the SAGE [17] and GALLEX [18 radiochemical experiments. These four observations, which probe different energy regimes in the solar neutrino spectrum, can all be simultaneously explained in terms of two flavor neutrino oscillations. The matter enhanced MSW [19 oscillation for a two flavor $\left(\nu_{e}-\nu_{x}\right)$ system admits two branches [20]: (i) The small angle non-adiabatic solution, which requires $\sin ^{2} 2 \theta=\left(3.5 \times 10^{-3}\right.$ to $1.5 \times$ $\left.10^{-2}\right)$ and $\Delta m^{2} \equiv\left(m_{2}^{2}-m_{1}^{2}\right)=\left(3.4 \times 10^{-6}\right.$ to $\left.1.2 \times 10^{-5}\right) e V^{2}$. (ii) The large angle MSW solution requiring $\sin ^{2} 2 \theta=(0.6-0.9)$ and $\Delta m^{2}=$ $\left(7 \times 10^{-6}\right.$ to $\left.5 \times 10^{-5}\right) e V^{2}$. Here $\nu_{x}$ could be $\nu_{\mu}, \nu_{\tau}$ or a sterile neutrino $\nu_{s}$ for the small angle MSW solution, while $\nu_{x}=\left(\nu_{\mu}\right.$ or $\left.\nu_{\tau}\right)$ in the large 
angle MSW solution. There is also the $\left(\nu_{e}-\nu_{x}\right)$ vacuum oscillation solution [21] for $\nu_{x}=\left(\nu_{\mu}\right.$ or $\left.\nu_{\tau}\right)$, with $\sin ^{2} 2 \theta=(0.6-1.0)$ and $\Delta m^{2} \sim$ $10^{-10} e V^{2}$.

2. Atmospheric neutrino anomaly: The ratio of muon to electron neutrinos from atmospheric cascades measured by the Kamiokande 22] and the IMB [23] experiments appear to suggest a deficit of about a factor of 2 when compared to Monte Carlo simulations [24]. For sub-GeV neutrinos (i.e., neutrinos with energy less than $\sim 1 \mathrm{GeV}$ ), Kamiokande finds for this ratio of ratios (which is expected to be 1 in the absence of neutrino oscillations) $R=0.60_{-0.05}^{+0.06} \pm 0.05$ which is in good agreement with the IMB value of $R=0.54 \pm 0.05 \pm 0.12$. Recently Kamiokande has also reported results for the multi-GeV neutrinos 25] where the ratio is $R=0.57_{-0.07}^{+0.08} \pm 0.07$, in agreement with the sub$\mathrm{GeV}$ data. This apparent deficit of $\nu_{\mu}$ 's or the excess of $\nu_{e}$ 's can be attributed to either $\left(\nu_{\mu}-\nu_{\tau}\right)$ oscillations with $\sin ^{2} 2 \theta=(0.65-1.0)$ and $\Delta m^{2}=\left(5 \times 10^{-3}\right.$ to $\left.3 \times 10^{-2}\right) e V^{2}$, or to $\left(\nu_{e}-\nu_{\mu}\right)$ oscillations with $\sin ^{2} 2 \theta=(0.55-1.0)$ and $\Delta m^{2}=\left(7 \times 10^{-3}\right.$ to $\left.7 \times 10^{-2}\right) e V^{2}$. The resolution of the anomaly in terms of $\left(\nu_{\mu}-\nu_{s}\right)$ oscillations, where $\nu_{s}$ is a sterile neutrino, will run into difficulty with primordial nucleosynthesis calculations which require that [26] $\Delta \mathrm{m}^{2} \sin ^{2} 2 \theta \leq 1.6 \times 10^{-6} \mathrm{eV}^{2}$. (Note that the solar $\left(\nu_{e}-\nu_{s}\right)$ oscillation parameters satisfy this constraint.) Among the other atmospheric neutrino experiments, only Soudan II [27] sees an anomaly with a preliminary value of $R=0.69 \pm 0.19 \pm 0.09$. The Frejus 28 results are marginally in conflict with the combined Kamiokande 
and IMB data. Considering the low statistics of Frejus experiment compared with the high statistical significance of Kamiokande and IMB data, we shall ignore this marginal discrepancy in our theoretical discussions.

3. Hot component of dark matter: As already noted in the introduction, and further explained in the next section, a combined fit to the COBE data as well as the data on distribution of galaxies on the large and small angular scales is difficult to achieve within the cold dark matter scenario. The simplest consistent scenario requires a significant hot component (15-30)\% in the dark matter [13, 29, 30] leading to the C+HDM scheme, with neutrinos being the natural candidates for the hot component. The mass of the neutrino comprising the hot dark matter should be in the few eV range.

4. $\bar{\nu}_{\mu}-\bar{\nu}_{e}$ oscillation at Accelerators: Recently the LSND experiment at the LAMPF facility in Los Alamos has reported positive evidence for $\bar{\nu}_{\mu}-\bar{\nu}_{e}$ oscillations 31. If these results survive further scrutiny and there is independent verification (e.g, at the KARMEN experiment [32]), it certainly will have a strong impact on particle and nuclear physics as well as cosmology and astrophysics. The initial LSND data, if interpreted in terms of two-neutrino oscillations suggest a mixing probability of $P_{\bar{\nu}_{\mu} \rightarrow \bar{\nu}_{e}}=\left(0.34_{-0.18}^{+0.20} \pm 0.07\right) \%$, when oscillation constraints from KARMEN and BNL-E776 33] accelerator searches as well as reactor neutrino constraints from Bugey facility [34] are folded in. In 
the discussion that follows, we shall take into considerations the sensitivity of the LSND and other accelerator and reactor experiments in searching for $\bar{\nu}_{\mu}-\bar{\nu}_{e}$ oscillations, but we shall not demand that the theoretical scenarios discussed correctly reproduce the precise numerical values reported by the LSND collaboration. We feel that this position is justified at this time, especially in view of the fact that a different analysis of the LSND data has yielded considerably weaker limits on the mixing parameters [35]. We find it remarkable however, that independent of the LSND data, two of the three scenarios which can accommodate points (1)-(3) above, imply an observable signal at accelerator $\left(\bar{\nu}_{\mu}-\bar{\nu}_{e}\right)$ oscillation experiments.

\subsection{Theoretical Schemes:}

Let us focus on the solar, atmospheric and hot dark matter neutrinos, leaving out the LSND results which will not play a role in determining the viable scenarios. It is clear that the mass-splittings required for explaining these three observations do not overlap $\left(\Delta \mathrm{m}^{2} \sim 10^{-5} \mathrm{eV}^{2}\right.$ for solar neutrinos, $\Delta m^{2} \sim 10^{-2} e V^{2}$ for atmospheric neutrinos and $m_{\nu} \sim(1$ to few $) \mathrm{eV}$ for hot dark matter). Assuming that there are no additional light neutrinos, one concludes that the three neutrinos $\left(\nu_{e}, \nu_{\mu}, \nu_{\tau}\right)$ should be nearly degenerate in mass. (Note that we often identify a mass eigenstate through its dominant flavor. The respective masses are $m_{1}, m_{2}$, and $m_{3}$.) This leads us to our first scenario.

Scenario (i): Three nearly degenerate neutrinos: Assume that $\nu_{e}, \nu_{\mu}$ and $\nu_{\tau}$ have a nearly common mass of about (1 to 3 ) eV. Their masses are split 
by small amounts, such that $\Delta m_{12}^{2} \equiv\left(m_{2}^{2}-m_{1}^{2}\right) \sim 10^{-5} \mathrm{eV}^{2}$ and $\left|\Delta m_{23}^{2}\right| \equiv$ $\left|\left(m_{3}^{2}-m_{2}^{2}\right)\right| \sim 10^{-2} e V^{2}$. Phenomenological neutrino mass matrices that are consistent with these assumptions are easily constructed [36]. This scheme will account for the solar neutrino data via $\left(\nu_{e}-\nu_{\mu}\right)$ MSW oscillations and the atmospheric neutrino anomaly in terms of $\left(\nu_{\mu}-\nu_{\tau}\right)$ oscillations. The required mixing angles are free parameters and can be adjusted to the suggested values. The $\left(\nu_{e}-\nu_{\tau}\right)$ mixing angle can be arbitrarily small, but it can also be as large as about 0.05. According to this scenario, neutrinoless double beta decay $\left(\beta \beta_{0 \nu}\right)$ is at (or even above?) the present experimental limit (modulo nuclear matrix element uncertainties), and so that it can be ruled out if $\beta \beta_{0 \nu}$ is not observed in the near future.

Also according to this scheme the $\nu_{\mu}-\nu_{\tau}$ oscillation experiments at accelerators (CHORUS/NOMAD, E803 [37, 38]), as well as $\nu_{e}-\nu_{\mu}$ oscillation searches at reactors, should fail to find anything interesting. In both cases the relevant $\Delta m^{2} \lesssim 10^{-2} \mathrm{eV}^{2}$, which is not in the range accessible in these experiments. However, this statement is strictly true only to the extent that the leptonic mixing matrix is assumed to be unitary. Deviation from unitarity can produce oscillation signals, although there should be no spatial dependence. This situation can arise if the ordinary neutrinos mix with a neutral heavy lepton with mass on the order of the electroweak scale. Let us consider this in more detail.

For definiteness, let us assume that the three light neutrinos have an admixture of a heavy fourth generation neutrino with mass greater than 
$M_{Z}$. Writing

$$
\nu_{e}=\sum_{i=1}^{4} U_{e i} \nu_{i}
$$

and similarly for the weak eigenstates $\nu_{\mu}, \nu_{\tau}$, one sees that muon neutrinos produced in $\pi$ decays will be the weak eigenstates with the heavy $\nu_{4}$ component removed. The $\nu_{e}$ and $\nu_{\mu}$ states are no longer orthogonal, leading to an apparent oscillation probability given by [39]

$$
\begin{aligned}
P_{\nu_{e}-\nu_{\mu}} & =\left|\left(U_{e 1}^{*} U_{\mu 1}+U_{e 2}^{*} U_{\mu 2}+U_{e 3}^{*} U_{\mu 3}\right)\right|^{2} \\
& =\left|-\left(U_{e 4}^{*} U_{\mu 4}\right)\right|^{2} .
\end{aligned}
$$

Here we have set the $\Delta m^{2}$ s to zero since they are all $\lesssim 10^{-2} e V^{2}$ and thus negligible for accelerator neutrino experiments. If the mixing parameters $U_{e 4}, U_{\mu 4} \sim\left(\frac{1}{4}\right.$ to $\left.\frac{1}{5}\right)$, then the oscillation probability at the LSND experiment will be in the range of $(2-3) \times 10^{-3}$ with no position dependence. The data presented in Ref. 31 is consistent with such an interpretation, as can be inferred from the large $\Delta m^{2}$ region of their Fig. 3. Various other constraints on such leakage to a heavy fourth generation neutrino has been studied in 40, where it is shown that the best existing limit is from neutrino oscillation experiments. $\nu_{\mu}-\nu_{\tau}$ oscillation probability of $(2-3) \times 10^{-3}$ can also be achieved by a similar mechanism, but this may be more difficult to measure.

If the heavy neutrino is a standard model singlet, there are stringent limits from $Z$-boson decays which make the non-unitary oscillations unobservable at accelerators. To see this, consider the mixing of ordinary neutrinos with a heavy isospin singlet neutral lepton $N$ of mass $m$. In the presence of such 
mixings, the invisible decay width of $Z$ will be modified so that the number of effective neutrino species coupling to $Z$ is given by

$$
N_{\nu}=3+\left(1-|\alpha|^{2}\right)\left[-\left(1+|\alpha|^{2}\right)+2 F|\alpha|^{2}+F^{\prime}\left(1-|\alpha|^{2}\right)\right]
$$

where $F$ and $F^{\prime}$ are phase space factors given by

$$
\begin{aligned}
F & =1-\frac{3}{2} x^{2}+\frac{1}{2} x^{3} \\
F^{\prime} & =\sqrt{1-4 x^{2}}\left(1-x^{2}\right)
\end{aligned}
$$

with $x \equiv m / m_{Z}$. It is to be understood here that for $m \leq m_{Z} / 2$, all terms in eq. (3) will contribute to $N_{\nu}$, while for $m \geq m_{Z}$, the $F$ and $F^{\prime}$ terms do not contribute. If $m_{Z} / 2 \leq m \leq m_{Z}$, the term proportional to $F^{\prime}$ should be set to zero, while $F$ is non-zero. The mixing parameter $|\alpha|$ is equal to the $(4,4)$ element of the unitary matrix $U_{\nu}$ which diagonalizes the neutrino mass matrix. It is related to the leptonic mixing matrix $U$ appearing in the charged current via

$$
|\alpha|^{2}=1-\left|U_{e 4}\right|^{2}-\left|U_{\mu 4}\right|^{2}-\left|U_{\tau 4}\right|^{2} .
$$

Although $U$, now a $3 \times 4$ matrix is not unitary, the $\nu_{e}-\nu_{\mu}$ transition probability is still given by eq. (2), since each row of $U$ is normalized to 1. It becomes apparent from eq. (2) and (5) that $P_{\nu_{e}-\nu_{\mu}} \leq\left(1-|\alpha|^{2}\right)^{2} / 4$. If the mass of $N$ is greater than $m_{Z}$, then $F=F^{\prime}=0$, so that $P_{\nu_{e}-\nu_{\mu}} \simeq$ $\left[\left(3-N_{\nu}\right) /\left(1+|\alpha|^{2}\right)\right]^{2} / 4 \equiv\left[\Delta N_{\nu} /\left(1+|\alpha|^{2}\right)\right]^{2} / 4 . \Delta N_{\nu}$ can be as large as 0.042 at 1 sigma 41], which when combined with $|\alpha|^{2} \simeq 1$ yields $P_{\nu_{e}-\nu_{\mu}} \leq 1.1 \times 10^{-4}$, too small to be observable. (Even with $\Delta N_{\nu}=0.067$, the 2 sigma value, $P_{\nu_{e}-\nu_{\mu}} \leq 2.8 \times 10^{-4}$.) If the mass of $N$ is less than $m_{Z} / 2$, then both the $F$ and 
$F^{\prime}$ terms in eq. (3) will be relevant, making $N_{\nu}$ more consistent with 3 . (As $F, F^{\prime} \rightarrow 1, N_{\nu} \rightarrow 3$.) However, such a scenario is ruled out by direct search limits for a heavy neutral lepton decaying into the usual charged leptons $(e, \mu)$, as should happen here. (The limit on the mass of such a neutral lepton is $m \geq 46 \mathrm{GeV})$.) If the mass of $N$ obeys $m_{Z} / 2 \leq m \leq m_{Z}$, then $F \neq 0$, but $F^{\prime}=0$ in eq. (3). In this case, noting that $F$ is at most $11 / 16$ (for $x=1 / 2)$, we see that $P_{\nu_{e}-\nu_{\mu}}$ can be as large as $\left(4 \Delta N_{\nu} / 5\right)^{2} \simeq 10^{-3}$, which is close to present experimental sensitivity. However, since the branching ratio $\operatorname{Br}[Z \rightarrow(N \bar{\nu}+\bar{N} \nu)] \sim 2 \times 10^{-4}$, few hundred such events should have been observed at LEP with the $N$ decaying subsequently into $l^{+} l^{-} \nu$. This possibility is also excluded based on the non-observation of such events.

We conclude that the case of isosinglet neutrino does not lead to any observable deviation from unitarity in the accelerator neutrino oscillation experiments. Note that there is no such constraint on a sequential fourth generation neutrino (or neutrinos in vector-like families).

Scenario (ii): No degenerate neutrinos: If there is no degeneracy in the neutrino masses and we assume some kind of a mass hierarchy, accounting for the solar, atmospheric and hot dark matter neutrinos would require the introduction of a light (sterile) neutrino $\nu_{s}$. There is then just one consistent scheme in this case. As far as we are aware, this possibility has not been discussed in the literature, so we shall elaborate on it.

By assumption, in this scenario the known neutrinos are non-degenerate, and indeed have hierarchical masses. The solar neutrino puzzle is resolved via $\left(\nu_{e}-\nu_{s}\right)$ MSW oscillations such that $m_{\nu_{s}}^{2}-m_{\nu_{e}}^{2} \sim 10^{-5} \mathrm{eV}$. The atmospheric 
neutrino anomaly is explained via $\left(\nu_{\mu}-\nu_{e}\right)$ oscillations with $m_{\nu_{\mu}} \sim 10^{-1} \mathrm{eV}$. The tau neutrino with a few eV mass constitutes the 'hot' component of dark matter.

As far as $\left(\bar{\nu}_{\mu}-\bar{\nu}_{e}\right)$ oscillations at accelerators are concerned, the "direct" transition is not possible because $\Delta m^{2} \sim 10^{-2} \mathrm{eV}^{2}$. However, it has recently been pointed out [42] that "indirect" $\left(\bar{\nu}_{\mu}-\bar{\nu}_{e}\right)$ transition via a virtual $\bar{\nu}_{\tau}$ in the few eV mass range can still occur in such a scheme. For this to be in the experimental reach, both $\left(\nu_{e}-\nu_{\tau}\right)$ oscillations (at reactors) and $\left(\nu_{\mu}-\nu_{\tau}\right)$ oscillations (at accelerators) should be in the observable range. As shown in [42], the present limits from Bugey reactor [34] on $\nu_{e}-\nu_{\tau}$ oscillations, and the CHARM-II, Fermilab E531 and CDHS limits [43] on $\left(\nu_{\mu}-\nu_{\tau}\right)$ oscillations translates into an observable "indirect" $\left(\nu_{e}-\nu_{\mu}\right)$ oscillations for a $\nu_{\tau}$ mass in the few eV range, which can account for the LSND results.

Let us point out that in this second scenario, the allowed MSW parameter region will be somewhat shifted because of the rapid $\left(\nu_{e}-\nu_{\mu}\right)$ oscillations from the sun to earth, leading to a further depletion of $\nu_{e}$ 's by a factor of $\frac{1}{2} \sin ^{2} 2 \theta_{e \mu} \sim\left(\frac{1}{4}\right.$ to $\left.\frac{1}{2}\right)$. This prediction will be tested in the forthcoming solar neutrino experiments.

This scheme may be on a somewhat better theoretical footing than the first one because no mass degeneracy is assumed. A light 'sterile' neutrino state, following 44, may arise from the hidden sector of some fundamental theory. If the hidden sector contains $S U(3) \times S U(2) \times U(1)$, one can define an unbroken 'parity' at the Lagrangian level. The $\nu_{R}$ 's needed for the seesaw mechanism, having no gauge quantum numbers can freely mix from the 
observable and hidden sectors. This would lead to mixing between the light neutrinos and their mirror partners (which are also light). Gravitationally induced interactions could also mix the neutrinos from the two sectors.

Scenario (iii): Nearly degenerate $\left(\nu_{\mu}-\nu_{\tau}\right)$ pair: This scheme also requires a light sterile neutrino $\nu_{s}$. In addition, an approximate lepton number symmetry such as $L_{e}+L_{\mu}-L_{\tau}$ is necessary to make $\nu_{\mu}$ and $\nu_{\tau}$ nearly degenerate in mass [36, 44, 45]. When this symmetry breaks by a small amount, maximal $\left(\nu_{\mu}-\nu_{\tau}\right)$ mixing occurs, facilitating a resolution of the atmospheric neutrino problem. The solar neutrino puzzle is explained via $\left(\nu_{e}-\nu_{s}\right)$ oscillations. The common mass of $\nu_{\mu}$ and $\nu_{\tau}$ is assumed to be $\sim$ few $\mathrm{eV}$, so that they constitute a 'two-flavor' hot dark matter. For accelerator neutrinos, there is direct $\left(\nu_{e}-\nu_{\mu}\right)$ oscillations at an observable level. In particular, the LSND data can be accommodated. However, $\left(\nu_{\mu}-\nu_{\tau}\right)$ oscillations will be beyond the reach of CHORUS/NOMAD and E803.

Aside from the two-flavor hot dark matter component, this scenario has other special cases relevant for cosmology. The solar neutrino data requires the mass splitting $\Delta m^{2}$ between $\nu_{e}$ and $\nu_{s}$ to be $\sim 10^{-5} \mathrm{eV}^{2}$, but the masses themselves may be in the cosmologically interesting range of a few eV. Since this pair behaves like Dirac neutrinos, there is no conflict with $\beta \beta_{0 \nu}$ constraints. For cosmology however, the hot dark matter may be a linear combination of $\nu_{e}$ and $\left(\nu_{\mu}+\nu_{\tau}\right)$. If the total mass of $\left(\nu_{\mu}, \nu_{\tau}\right)$ pair exceeds that of $\nu_{e}$, we call it a $(2+1) \mathrm{C}+\mathrm{HDM}$ scheme, and if the $\nu_{e}$ mass dominates over $\left(\nu_{\mu}, \nu_{\tau}\right)$, it is a $(1+2)$ scheme. Of course, one could also recover the one flavor case by making the mass of $\left(\nu_{\mu}-\nu_{\tau}\right)$ pair much smaller than $1 \mathrm{eV}$. 
(This case will correspond to an inverted mass hierarchy 46.)

\section{Cosmological Implications}

In studies of large scale structure, it has become clear that a critical density Cold Dark Matter (CDM) universe with density perturbations that have roughly a Harrison-Zeldovich spectrum cannot simultaneous fit observations of structure on large and small scales. Normalized on large scales to fit the COBE observations, CDM produces too many clusters of galaxies and galactic pairwise velocities far in excess of observations 47. This basic problem is neatly resolved in a cold plus hot dark matter scenario because the growth of the density perturbations on small scales is damped by the presence of the hot dark matter component [1, 7, 11]. Since the neutrino oscillation experiments may be telling us that the neutrino component is spread among several flavors we would like to understand, in a quantitative way, how this changes the usual (one flavor) cold plus hot dark matter model. We will begin with a general description of the effects of a hot component on the growth of density fluctuations. First we will give some analytic formulae for estimating the size of the effects, then we will show some results of more detailed calculations. Finally we conclude with some remarks about the uncertainties in cosmological parameters which currently prevent one to use cosmological data to pin down the values of the neutrino masses. 


\subsection{Free-Streaming or Jeans Masses}

Neutrinos retain large velocities from the time before primordial nucleosynthesis when they were in thermal equilibrium. This means that if one initially sets up density perturbations, the neutrinos will rearrange themselves to a different pattern at some later time, erasing neutrino density fluctuations on length scales over which the neutrinos can have traveled. This "freestreaming" or "Jeans" length scale $\lambda_{J}$ is given at time $t$ by

$$
\lambda_{J}(t)=a(t) \tau(t) v(t)
$$

where $a(t)$ is the scale factor which describes the expansion of the universe, $\tau(t)$ is the conformal time $(d \tau=d t / a(t))$, so $a(t) \tau(t)$ is the physical horizon size, and $v(t)$ is the average neutrino velocity. An analytic fit for $v$ is given by 48

$$
v(t)=\left[1+\left(\frac{a}{a_{n r}}\right)^{2}\right]^{-1 / 2},
$$

where $a_{n r}$ is the scale factor when the neutrinos become non-relativistic, and we use units where the velocity of light is unity. We have

$$
a_{n r}=2.25 T_{\gamma}^{0} / m_{\nu}
$$

where $T_{\gamma}^{0}=2.35 \times 10^{-4} \mathrm{eV}$ is the present cosmic background photon temperature. The value of the Jeans' length at the current time $t_{0}$ is

$$
\lambda_{J}\left(t_{0}\right)=3 t_{0} a_{n r}=3.2\left(\frac{1 \mathrm{eV}}{m_{\nu}}\right) h^{-1} \mathrm{Mpc}
$$

$h$ is the parametrization of our ignorance of the true value of the Hubble constant $H_{0} \equiv 100 h \mathrm{~km} \mathrm{~s}^{-1} \mathrm{Mpc}^{-1}$. To relate this length to the masses of 
known astrophysical structures we convert to the total mass contained in the volume $4 \pi\left(\lambda_{J} / 2\right)^{3} / 3$, (the Jeans' mass). Currently, the Jeans mass $M_{J_{\nu}}\left(t_{0}\right)$ is

$$
M_{J_{\nu}}\left(t_{0}\right)=M_{H_{0}}\left(a_{n r}\right)^{3}=4.6 \times 10^{12}\left(\frac{1 \mathrm{eV}}{m_{\nu}}\right)^{3} h^{-1} M_{\odot}
$$

where $M_{H_{0}}=3.14 \times 10^{22} h^{-1} M_{\odot}$ is the mass contained in the current Hubble volume. Interestingly enough, the mass in (10) is comparable to a galactic mass for neutrinos in the range $m_{\nu} \simeq 1-4 \mathrm{eV}$. Note that in the formulae presented here we assume a critical density of matter, and only a fraction is in the form of hot dark matter- the remainder is comprised of cold dark matter and baryons.

During the matter dominated epoch, $\rho \propto a^{-3}, v \propto a^{-1}$ (while the neutrinos are non-relativistic) and $a \tau \propto a^{3 / 2}$, so the Jeans' mass $M_{J \nu} \propto a^{-3 / 2}$ and decreases with time. When the neutrinos are relativistic, $v \sim 1$, and the Jeans' mass increases with time. The maximum in the Jeans mass occurs when the neutrinos are just becoming non-relativistic, i.e., when $a(t)=a_{n r}$ :

$$
M_{J \nu}(\max ) \simeq M_{H_{0}}\left(a_{n r}\right)^{3 / 2}=4 \times 10^{17}\left(\frac{1 \mathrm{eV}}{m_{\nu}}\right)^{3 / 2} h^{-1} M_{\odot} .
$$

This relation is approximate, because the time when neutrinos become relativistic is quite close to the time when matter begins to dominate over radiation in the universe, so the horizon size does not scale exactly as $a^{-3 / 2}$. The exact formula must be determined numerically for each value of the neutrino mass. The formula in (11) is accurate to within an order of magnitude, which is good enough for estimating the size of the effects under study here. In the older models of structure formation in which the dark matter is to- 
tally composed of neutrinos of mass $\sim 30 \mathrm{eV}$, this maximum Jeans mass is somewhat larger than a typical mass of a cluster of galaxies.

In studies of single massive flavor $\mathrm{C}+\mathrm{HDM}$ models, a more typical value of the neutrino mass $\sim 6 \mathrm{eV}$ (for $h=0.5$ ) was preferred, yielding a maximum Jeans' mass of $M_{J_{\nu}}(\max )=3 \times 10^{16} h^{-1} M_{\odot}$, which is slightly smaller than the so-called "great attractor" [49], the largest concentration of mass which is pulling galaxies gravitationally on scales of order $40 h^{-1}$ Mpc. If we assume, as in scenario i), that the same $\Omega_{\nu}$ is now composed of 3 nearly degenerate neutrinos of mass $\sim 2 \mathrm{eV}, M_{J_{\nu}}(\max )=1 \times 10^{17} h^{-1} M_{\odot}$, which is somewhat larger than the "great attractor" mass.

We will proceed to examine how these two mass scales $\left(M_{J_{\nu}}\left(t_{0}\right)\right.$ and $\left.M_{J \nu}(\max )\right)$ are incorporated in predictions of structure formation.

\subsection{Growth of Density Perturbations}

In the early universe, when matter begins to dominate the energy density of the universe, the CDM and C+HDM mass fluctuation spectra are identical. The only feature imprinted on the initial spectrum at this time is a decrease of amplitude on length scales smaller than the horizon at matter

domination. These scales experience a smaller amount of growth during the radiation dominated era due to the effects of radiation pressure. After matter domination the fluctuation amplitudes on these scales can grow, driven by their own self-gravitation. If a volume of space contains a mass $\tilde{M}$ which is slightly different than the average $M$ for that size, and the dark matter is strictly CDM, the fluctuation amplitude will grow proportional to the scale 
factor $a$

$$
\frac{\delta M}{M}(M) \equiv \frac{\tilde{M}-M}{M} \propto a
$$

Growth in the $\mathrm{C}+\mathrm{HDM}$ model is more interesting. When a mass fluctuation contains a mass $M<M_{J_{\nu}}(t)$ at time $t$, the neutrinos will stream out of the fluctuation and change the local gravitational potential. This smaller potential also reduces the growth rate of the mass fluctuations to [1, 50]

$$
\frac{\delta M}{M}(M) \propto a^{p} ; p=\frac{1}{4}\left(-1+\sqrt{25-24 \Omega_{\nu}}\right)
$$

The fact that the Jeans mass decreases with time means that if $M<$ $M_{J_{\nu}}$ at some early time, sometime in the future $M>M_{J_{\nu}}$ will be true. Once $M>M_{J_{\nu}}$, the growth rate returns to the CDM growth rate, as the neutrinos will then effectively be "cold" on this mass scale. This leads to scale dependent changes in the mass fluctuation spectrum. The equations for the Jeans mass (11) and (10), and the growth rate (13) also illustrate the effect of having multiple flavors of massive neutrinos with comparable masses. The Jeans mass depends on the individual neutrino mass, while the growth rate depends only on the sum of the neutrino masses, as

$$
\Omega_{\nu}=h^{-2} \sum_{i=e, \mu, \tau} \frac{m_{\nu_{i}}}{94 \mathrm{eV}}
$$

So increasing the number of degenerate flavors means that the Jeans mass will be increased for a given value of $\Omega_{\nu}$.

The growth of fluctuations on mass scale $M$ in the intermediate region $M_{J_{\nu}}(\max )<M<M_{J_{\nu}}($ now $)$ will be "damped" relative to their value in a universe dominated only by CDM. The amount of damping between the 
time when the neutrinos become non-relativistic and when the Jeans mass becomes smaller than scale $M$ is given by

$$
\text { damping factor }=\left[\frac{M_{J_{\nu}}(\max )}{M}\right]^{(2 p-2) / 3}
$$

The maximum amount of damping occurs for mass scales $M \ll M_{J \nu}\left(t_{0}\right)$ which is a constant given by

$$
\text { max damping factor }=\left[\frac{M_{J_{\nu}}(\max )}{M_{J_{\nu}}\left(t_{0}\right)}\right]^{(2 p-2) / 3}=\left(a_{n r}\right)^{1-p}
$$

The difference, then, between having the mass equally spread among $\mathcal{N}_{\nu}$ flavors as opposed to 1 flavor results in an additional small scale damping of $\mathcal{N}_{\nu}^{1-p}$, which is between $10 \%-20 \%$ for $0.20<\Omega_{\nu}<0.30$.

The combination of hot and cold components of dark matter thus has the following effects, when compared to the a model with only a cold component. First of all, the growth of density perturbations with masses in the intermediate range $M_{J}($ now $)<M<M_{J}(\max )$ is reduced by the factor given in eq. (15) which depends mainly on $\Omega_{\nu}$. Below $M_{J}($ now) the mass fluctuation spectrum follows the scale dependence of a CDM model but with an amplitude reduced by the factor in eq. (16). Splitting the neutrino density among $\mathcal{N}_{\nu}$ flavors of approximately degenerate neutrinos produces an addi-

tional damping of $\mathcal{N}_{\nu}^{p-1}$ below a somewhat higher value of $M_{J \nu}(\max )$. This additional damping may be important for reconciling observations of clusters of galaxies with a COBE normalized spectrum of density fluctuations.

\subsection{Detailed Comparisons to Structure Formation}

In order to do a more careful comparison of these models to observed large scale structure, a more accurate evaluation of the effects expressed above 
is required. We achieve this by integrating the linearized general relativistic evolution equations for the photons, neutrinos (massive and effectively massless flavors), baryons and CDM particles. The procedure we use is described in detail in ref. [51]. The models have a baryon mass fraction $\Omega_{\text {baryon }}=0.0125 / h^{2}$, which is reasonably consistent with nucleosynthesis [52]. The CDM fraction is then given by $\Omega_{C D M}=1-\Omega_{\nu}-\Omega_{\text {baryon }}$ (since here we are only considering critical density universes). In figures 1 and 2 , we use a Harrison-Zeldovich (scale free) spectrum normalized to COBE [53 with $h=0.5$. After smoothing the density field with a low pass Gaussian filter, we arrive at the curves shown in figures 1 and 2. The masses on the X-axis are $(2 \pi)^{3 / 2} r_{f}^{3} \rho$, as appropriate for a Gaussian filter radius of $r_{f}$. In the three panels a), b), and c), we show curves for $\Omega_{\nu}=0.20,0.25$, and 0.30 respectively. Within each panel we show curves for 1, 2, and 3, degenerate neutrino flavors. In panel a), we also display the analogous CDM model curve for comparison.

The models under consideration are now known to fit a large amount of data on large scale structure [6, 12, 13, 29], but here we will concentrate on the two toughest constraints for $\mathrm{C}+\mathrm{HDM}$ models to satisfy. The first constraint is on the amplitude of mass fluctuations which form clusters. It has been argued [54 that the number density of rich clusters requires that on a scale $\sim 10^{15} M_{\odot}, \delta M / M=0.58 \pm 0.1$ (2 times their "error bar"). As stated in ref. [54 the errors are hard to estimate because of possible systematic uncertainties in the masses assigned to clusters. Indeed, the mass scales for two different determinations of the cluster mass function in refs [55] and 
[56] differ by almost a factor of 2. Ref. [54 argues that the cluster masses could be overestimated, while measurements of the gravitational lensing by the clusters suggest [59] that the usual mass estimates (used to determine $(\delta M / M))$ may be low by as much as a factor of 3 . In order to allow for this uncertainty, we also give an estimated horizontal error bar which is a factor of two (in cluster mass) from the value $1 \times 10^{15} h^{-1} M_{\odot}$, to reflect the size of the possible systematic error.

At the far left end of the figures we have also drawn a line which corresponds to the lower limit of the mass fluctuation amplitude implied by the measurements of the abundance of "damped Lyman alpha systems" as indicated by the recent survey of ref. 60]; see also ref. [61]. While early work 62 indicated that $\mathrm{C}+\mathrm{HDM}$ models (with $\Omega_{\nu}=0.3$ ) predictions were far below the observations, it now seems as though the assumptions used may have been too restrictive. There is little information about the exact nature of these damped Lyman alpha systems, other than their neutral hydrogen column densities and spectral line widths. A more general analysis 63 shows how to properly estimate number abundances of objects on these scales [64]. We have used this latter procedure to estimate the amplitude of the constraint line in figures 1 and 2. It is still uncertain what mass is to be identified with these damped Lyman alpha systems. It seems reasonable that we should associate dark matter halo masses of between $\sim 10^{10}-10^{11} h^{-1} M_{\odot}$ and we have placed our constraint line there in our figures. (The curves should pass above the constraint line somewhere in the range.) The mixed dark matter models are more compatible with the lower end of this mass range. However, 
with $\Omega_{\nu}=0.30$ and $h=0.5$ one has to take very small halo masses even to make this model work marginally. From figure 1 then it is apparent that $\Omega_{\nu}<0.30$ if $h=0.5$ with a pure Harrison-Zeldovich spectrum.

Figure 1 also points out an interesting aspect of spreading the neutrino mass density among a number of massive neutrino flavors $\mathcal{N}_{\nu}$. With $\mathcal{N}_{\nu}=1$, the amplitude of the density fluctuations on cluster scales seems to be slightly larger than what is required; increasing $\mathcal{N}_{\nu}$ improves the fit. This result depends only weakly on $\Omega_{\nu}$ on this mass scale. [Eq.(15) predicts less than $10 \%$ difference in the cluster scale $\delta M / M$ amplitude over the range $\Omega_{\nu} \sim 0.2-0.3$.]

However, because of the $\Omega_{\nu}$ dependence in the $\mathcal{N}_{\nu}^{p-1}$ damping factor (via $p$ ), $\mathcal{N}_{\nu}=2$ works best for $\Omega_{\nu}=0.30$ and $\mathcal{N}_{\nu}=3$ works best for $\Omega_{\nu}=0.20$. On the other hand increasing the number of flavors makes the disagreement with Lyman alpha systems datum worse for $\Omega_{\nu}=0.30$, so $\mathcal{N}_{\nu}>1$ seems to work best for models with $\Omega_{\nu}<0.30$.

We should point out here that the conclusions we draw from figure 1 hold only for the spectrum, Hubble constant and baryon fraction used in the models, and altering any of these parameters changes the conclusions. We will explore this further later. The purpose of figure 1 is mainly to illustrate the effects of modifying the hot dark matter composition.

\subsection{Additional Models Which Can Accommodate the LSND Results}

In the above we have considered the consequences of the three neutrino scenarios in which we have $\mathcal{N}_{\nu}$ flavors with roughly equal mass. However, if we take the LSND results as a serious constraint, then scenario (iii) admits the 
possibility that all three known neutrino flavors can have masses in the $\mathrm{eV}$ range, although the electron neutrino mass can still be significantly different from the $\mu$ and $\tau$ neutrino masses (which form a degenerate pair). Since only the square of the mass difference is measured, we have two cases to consider: a) $m_{\nu_{\mu}}>m_{\nu_{e}}$, ("2+1") and b) $m_{\nu_{e}}>m_{\nu_{\mu}}$ ("1+2").

In a " $2+1$ " model, where $\nu_{\mu}$ and $\nu_{\tau}$ are each heavier than $\nu_{e}$, the lightest neutrino $\left(\nu_{e}\right)$ will have a very large maximum Jeans' mass, $\left(M_{J_{\nu}}^{l}(\max )\right)$, compared to the other two heavier neutrino Jeans masses $\left(M_{J_{\nu}}^{h}(\max )\right)$. In the " $1+2$ " model, where $\nu_{\mu}$ and $\nu_{\tau}$ are lighter than $\nu_{e}$, the lightest neutrinos $\left(\nu_{\mu}\right.$ and $\left.\nu_{\tau}\right)$ will have very large $M_{J_{\nu}}^{l}(\max )$, compared to the remaining heavier $\nu_{e}$ neutrino $M_{J_{\nu}}^{h}(\max )$. For $M_{J_{\nu}}^{l}(\max )>M>M_{J_{\nu}}^{h}(\max )$, there will be an amount of damping which would be there if only the lightest neutrino comprised $\Omega_{\nu}$. For $M_{J_{\nu}}^{h}(\max )>M$ the damping will be intermediate between the one and three flavor massive neutrino case.

To illustrate these two cases, we will use values of $\Delta m^{2}=8$ and $20 \mathrm{eV}^{2}$, for the " $2+1$ " and " $1+2$ " models respectively in the context of an $\Omega_{\nu}=0.25$, $h=0.5, n=1$ cosmology. In figure 2 we plot the curves for the $2+1$ model $\left(m_{\nu_{e}}=0.22 \mathrm{eV}\right.$ and $\left.m_{\nu_{\tau}}, m_{\nu_{\mu}}=2.84 \mathrm{eV}\right)$, and the $1+2$ model $\left(m_{\nu_{e}}=4.524\right.$ $\mathrm{eV}$ and $m_{\nu_{\tau}}, m_{\nu_{\mu}}=0.685 \mathrm{eV}$. For comparison we also plot the curves for $\mathcal{N}_{\nu}=1,2$, and 3 flavors for the same $\Omega_{\nu}(=0.25)$. The curve for $2+1(1+2)$ lies in between the curves for the 2 and 3 ( 1 and 2) degenerate flavors.

We can estimate the difference between having 3 flavors with equal and unequal masses as follows. In the " $1+2$ " and " $2+1$ " models the lightest flavor mass $m_{\nu l}$ is lighter than the three equal flavor mass $m_{\nu 3}$. The heavier 
flavor mass $m_{\nu h}$ is greater than $m_{\nu 3}$. Consider a mass scale $M<M_{J_{\nu}}^{h}(\max )$. After some time, when $M_{J_{\nu}}^{h}(t)=M$, the amount of damping is the same in both the equal and unequal mass cases. After that time that scale will experience an additional damping of $\left[M_{J_{\nu}}^{l}(\max ) / M_{J_{\nu}}^{h}(\max )\right]^{\left(2 p_{l}-2\right) / 3}$ (eq. 15) in the unequal mass case, and $\left[M_{J_{\nu}}^{(3 \text { flavors })}(\max ) / M_{J_{\nu}}^{h}(\max )\right]^{(2 p-2) / 3}$ in the 3 flavor case. Here $p_{l}$ is $p$ in equation (13) calculated assuming $\Omega_{\nu}$ is only made from the lightest neutrino(s). The ratio of the damping in the $1+2$ (and $2+1$ ) cases to the 3 equal mass case is (for scales $<M_{J_{\nu}}^{h}(\max )$ )

$$
\text { damping ratio }=\frac{\left(m_{\nu l} / m_{\nu h}\right)^{\left(1-p_{l}\right)}}{\left(m_{\nu 3} / m_{\nu h}\right)^{(1-p)}},
$$

The ratio in (17) is always larger than 1, indicating that the damping is greatest in the 3 equal flavor case. However, if the ratio of the masses of heavy to light flavors is $\lesssim 4$ the difference between damping in the various cases differs from the 3 flavor case by $<2 \%$. This is the reason for choosing the particular $\Delta m^{2}$ for plotting. Had we plotted, for example, the $2+1$ and $1+2$ models using $\Delta m^{2}=6 \mathrm{eV}^{2}$, (the same value used in ref. [4], but for $\left.\Omega_{\nu}=0.20\right)$ both of the curves would be nearly indistinguishable from the 3 degenerate neutrino case for masses $<M_{J_{\nu}}^{h}(\max )$. When the light and heavy masses differ by an order of magnitude or more, as in figure 2, the $2+1$ model goes to the 2 degenerate flavor model and the $1+2$ goes to the single flavor model. We note that because the lightest neutrino weighs less than the 3 degenerate flavor neutrino, on the very largest scales where $M_{J_{\nu}}^{l}(\max )<M<M_{J_{\nu}}^{(3 \text { flavors })}(\max )$, there will be some damping which is absent in the 3 degenerate flavor case.

We see that these additional models offer now a continuum of $\Delta M / M$ 
values between the 1 and 3 degenerate mass neutrino cases. The sole motivation for considering a case where the neutrinos have masses and mass differences of order a few $\mathrm{eV}$ in scenario iii) is the possibility of detecting oscillations with $\Delta m^{2}$ in the few $\mathrm{eV}^{2}$ range in the LSND (and also KARMEN) experiments. In the framework of GUT theories however, such a mass spectrum will not be easy to understand.

\subsection{Sensitivity to Cosmological Parameters}

In the previous sections we have plotted results for specific values of the Hubble constant, initial density fluctuation spectrum, and $\Omega_{\text {baryon }}$. We have not drawn strong conclusions about the neutrino mass scenarios, because the results are quite sensitive to the specific values used for these cosmological parameters. We will discuss the effects of these parameters on the cosmological structure formation and give specific examples of models from each of the three mass scenarios.

1. $H_{0}$ : The value of the Hubble constant has been the subject of a long and ongoing observational campaign (see 65 for some recent measurements). The experiments find that $0.4<h<1.0$. This uncertainty is

quite large when seen from the point of view of the required neutrino masses. Rewriting equation (14) for degenerate neutrinos we find

$$
m_{\nu}=4.7 \frac{1}{\mathcal{N}_{\nu}} \frac{\Omega_{\nu}}{0.2}\left(\frac{h}{0.5}\right)^{2} \mathrm{eV}
$$

which emphasizes that the neutrino masses scale as $h^{2}$. In figures 1 and 2 we have used $h=0.5$. Some of the recent measurements [65] seem 
to imply a larger value of $h$. In particular, if $h=0.6$, then the masses used in the previous examples are increased by a factor of 1.44. For example, ref. [4] advocates using $\mathcal{N}_{\nu}=2$ and $\Omega_{\nu}=0.2$ with $\Delta m^{2}=6$ $\mathrm{eV}^{2}, h=0.5$. If instead $h=0.6$ with the other parameters fixed, then the oscillation signal at Los Alamos would be consistent with $\Delta m^{2}=12$ $\mathrm{eV}^{2}$.

The scale which corresponds to the horizon size at matter domination also depends on the Hubble constant. If we use a larger value of $h$, then matter domination occurs earlier, when the horizon size was smaller, which means that the radiation dominated era is less effective at arresting the growth of small scale fluctuations. Such an effect exacerbates the small scale problems in CDM models and favors using a larger value of $\Omega_{\nu}[3,30]$.

are attributed

\section{Initial Mass Fluctuation Spectrum}

It has long been known that inflation predicts a power spectrum of density fluctuations with a spectral index $n$ close to unity. Since the amount of deviation is strongly model dependent, many investigators are content to use $n=1$. In figures 1 and 2 we have also used the value of $n=1$ for the spectral index. However, even small deviations from $n=1$ can lead to significant changes in the conclusions. To see this we give the initial spectrum in terms of rms mass fluctuations $\left(\frac{\Delta M}{M}\right)$ :

$$
\frac{\Delta M}{M} \propto M^{-(3+n) / 6}
$$


In the simplest models of inflation, particularly those based on GUTs (see, e.g., ref. [29, 30]), $n \sim 0.94-0.98$, although other values of $n$ are certainly possible [66]. From eq. (19) we see that decreasing (increasing) $n$ from unity decreases (increases) the small scale power. Since the models are normalized at large scales to COBE observations the mass fluctuation curves in figures 1 and 2 will "pivot" around a very large mass scale $\sim 10^{21} h^{-1} M_{\odot}$. The "pivot" mass scale here is somewhat smaller than the horizon mass, because the best fit COBE quadrupole anisotropy scales as $e^{1-n}$ 53. (We have fit our spectra by normalizing to the 7th multipole moment of the Sachs-Wolfe anisotropy, as recommended in ref. [53])

3. $\Omega_{\text {baryon. }}$ In the past few years comparisons of the primordial light element abundances inferred from observations with those calculated have led to strict limits [52 on the amount of baryonic material in the universe, $0.04<\Omega_{\text {baryon }}(0.5 / h)^{2}<0.06$. We have used the central value of this range for the models in figures 1 and 2. Improvements in the observations of deuterium and ${ }^{3} \mathrm{He}$ and determinations of the neutron lifetime have now led to a situation where all of the light element abundances are not consistent in a universe where the three known neutrino flavors have the standard number densities (derived from thermal equilibrium) at nucleosynthesis 67. If, however, the ${ }^{4} \mathrm{He}$ abundances derived from observations have been systematically underestimated 68 by about 5\%-10\% then big bang nucleosynthesis would be made consistent with the three flavors of neutrinos, provided that the baryon 
density is about $50 \%$ larger than previously thought. This would go in the right direction to explain why the baryon to dark matter ratio in cluster cores is so much larger than $\Omega_{\text {baryon }}$ [69].

If we allow for larger baryon fractions, this will also change the amplitude of the mass fluctuations for a given $\Omega_{\nu}$ and $n$. We can understand this through the following. Baryons (mostly protons) after nucleosynthesis are electrically charged and so are strongly coupled to the photon field. Baryonic density fluctuations cannot grow until the photon temperature decreases to allow the stable formation of neutral hydrogen. Because photon-baryon decoupling does not happen until after matter domination, the baryons are prevented from falling into the gravitational wells supplied by the cold dark matter. This leads to a damping in the growth of density fluctuations (relative to a case with no baryons). The amount of damping (on scales smaller than the horizon size at photon baryon decoupling) in the final amplitude of density fluctuations is constant, so the effect is quite similar to changing the number of degenerate neutrino flavors.

These uncertainties mean that one cannot currently use the cosmological data to determine which of our three scenarios is correct, or even what the value of $\Omega_{\nu}$ is. One must do a systematic study of the available parameter space taking into account the full range of these parameters. Studies of the interplay between these cosmological parameters have been done in various contexts [13, 29, 70, with more in progress [71. 
As a graphic illustration of this uncertainty, and also to demonstrate use of the formulae presented in sections 3.1-3.3, we show four models in figure 3 that give nearly identical predictions of structure formation, but use quite different solutions of the neutrino oscillation data. The choices of parameters are as follows.

1. The solid curve. [Scenario (iii)]. This model with $\mathcal{N}_{\nu}=2, \Omega_{\nu}=0.20$, $n=1$, and $h=0.5$ is advocated as a good fit to cosmological observations by ref. [- 1 consistent with the Los Alamos LSND experiment. We present this model for comparison.

2. The dot-dashed curve. [Also scenario (iii)]. We increase $h$ to 0.6 in this $\mathcal{N}_{\nu}=2$ model, but now there will be too much small scale power. To compensate for this, we increase $\Omega_{\nu}$ to 0.25 , and decrease $n$ to 0.95, a value which actually is more in line with standard inflationary predictions. The $\bar{\nu}_{e}-\bar{\nu}_{\mu}$ experiments would then be expected to see a signal corresponding to $\Delta m^{2}=18 \mathrm{eV}^{2}$. The baryon fraction has been scaled from the first model as $h^{2}$ consistent with the nucleosynthesis constraints.

3. The short dashed curve. [Scenario (i)]. Again using $h=0.6$ and $\Omega_{\nu}$, we now consider $\mathcal{N}_{\nu}=3$ degenerate flavors. Because the extra flavor provides an additional damping factor over the two flavor model of $(2 / 3)^{16}=0.94$, (eq. 16), to get a curve similar to the previous one we can increase $n$ to 0.98 , which increases the amplitude at the cluster mass by $\left(10^{21} / 10^{15}\right)^{0.0075}=1.10$, (eq. 19), so we get very nearly the 
same fit at the cluster scale. The value $n=0.98$ happens to be the value predicted in a particular SUSY GUT inflation model [30].

4. The long dashed curve. [Scenario (ii)]. Here we return to $h=0.5$, $\Omega_{\nu}=0.2$, and $n=1$ as in the solid curve. To compensate for the increase in amplitude by going back to 1 flavor $(2 / 1)^{0.13}=1.09$ in the solid curve, we increase $\Omega_{\text {baryon }}$ to 0.10 . This value of $\Omega_{\text {baryon }}$ is consistent with 3 relativistic neutrino flavors during nucleosynthesis if the ${ }^{4} \mathrm{He}$ abundances have been systematically underestimated.

We have now shown that allowing for more than one degenerate (in mass) neutrino state, as indicated by solar and atmospheric oscillation experiments, produces a "degeneracy" in the predictions of structure formation for $\mathrm{C}+\mathrm{HDM}$ models, given the uncertainty in cosmological parameters. In order to break this latter "degeneracy" we need a convincing detection of neutrino oscillations in an accelerator experiment, or an improvement in the determination of cosmological parameters, or both. In the meantime, we just note that there is a very rich structure contained in $\mathrm{C}+\mathrm{HDM}$ models of structure formation, which still allows considerable flexibility in fitting astrophysical data.

\section{Conclusions}

We have investigated the impact of neutrino oscillations, as indicated by a number of solar and atmospheric neutrino experiments, on the 'cold plus hot' dark matter scenario of large scale structure formation. We are led 
to three distinct scenarios for neutrino masses and mixings with interesting predictions for the ongoing/planned experiments. We note in particular the expectations for $\bar{\nu}_{\mu}-\bar{\nu}_{e}$ and $\nu_{\mu}-\nu_{\tau}$ oscillations being currently searched for. The cosmological implications of the three scenarios are explored in some detail. For some choices of the cosmological parameters (particularly $h=0.5$, $n=1$, and $\left.\Omega_{\text {baryon }}=0.05\right)$, the two and three neutrino flavors scenarios provide a somewhat better fit to the present data than the single (neutrino) flavor case. However, as we show, this need not hold for a different parameter choice. It is too early to pick out the best model but it is remarkable that taking account of the oscillation experiments, the $\mathrm{C}+\mathrm{HDM}$ models can still provide a good fit to the large scale structure data within the context of the simplest inflation models.

\section{References}

[1] Q. Shafi and F. W. Stecker, Phys. Rev. Lett., 53, 1292, (1984); Q. Shafi, NASA Technical Memorandum, May, (1993).

[2] R. Holman, G. Lazarides, and Q. Shafi, Phys. Rev. D, 27, 995, (1983); R. N. Mohapatra and G. Senjanovic, Z. Phys. C 17. 53, (1983).

[3] D. Pogosyan and A. Starobinsky, 11th Potsdam Workshop on Rel. Astrophys., Potsdam, (1994).

[4] J. R. Primack, J. Holtzman, A. A. Klypin, D. O. Caldwell, Phys. Rev. Lett., 74, 2160, (1995). 
[5] Note that 3 degenerate flavors was also considered in refs. [7], [8] and the last article in ref. [6].

[6] F. Occhionero and R. Scaramella, Astron. Astrop., 204, 3, (1988);

A. van Dalen and R. K. Schaefer, Astrophys. J., 398, 33, (1992); S. Xiang and T. Kiang, Mon. Not. Roy. Ast. Soc., 259, 761, (1992)

[7] R. Schaefer, Q. Shafi and F. W. Stecker, Astrophys. J., 347, 575, (1989).

[8] J. A. Holtzman, Astrophys. J. Suppl., 74, 1, (1989).

[9] G. F. Smoot, et al., Astrophys. J., 396, L1, (1992); E. L. Wright, et al., Astrophys. J., 396, L13, (1992).

[10] This point was highlighted in R. Schafer and Q. Shafi, Nature 359, 199 (1992).

[11] M. Davis, M. Summers, and D. Schlegel, Nature, 359, 393, (1992); A. Klypin, J. Holtzman, J. Primack, and E. Regos, Astrophys. J., 416, 1, (1993); Y. P. Jing, H. J. Mo, G. Börner, and L. Z. Fang, Astrophys. J, 411, 450, (1993); C.P. Ma and E.Berschinger, Astrophys. J.,, 434, L5, (1994).

[12] A. N. Taylor and M. Rowan-Robinson, Nature, 359, 396, (1992); R. K. Schaefer and Q. Shafi, Phys. Rev. D, 47, 1333, (1993); J. A. Holtzman and J. R. Primack, Astrophys. J., 405, 428, (1993); A. R. Liddle and D. H. Lyth, Mon. Not. Roy. Ast. Soc., 265, 379, (1993) 
[13] D. Yu. Pogosyan and A.A. Starobinski, Mon. Not. Roy. Ast. Soc., 265, 507, (1993).

[14] J.N. Bahcall, Neutrino Astrophysics, Cambridge University Press, Cambridge, 1989.

[15] For recent results, see R. Davis, in Frontiers in Neutrino Astrophysics, edited by Y. Suzuki and K. Nakamura (Universal Academy Press, Tokyo) p. 47 (1993).

[16] K.S. Hirata et. al., Phys. Rev. Lett. 65, 1297, 1301 (1990); Phys. Rev. D44, 2241 (1991).

[17] A.I. Abazov et. al., Phys. Rev. Lett. 673332 (1991).

[18] P. Anselman et. al., Phys. Lett. B285, 376 (1992); B314, 445 (1993); B327, 377 (1994).

[19] S.P. Mikheyev and A. Yu. Smirnov, Sov. J. Nucl. Phys. 42, 913 (1986); L. Wolfenstein, Phys. Rev. D17, 2369 (1978).

[20] For recent updates, see P. Langacker, Erice Summer School, 1994 (hepph/9411399); P.I. Krastev and S.T. Petcov, Preprint hep-ph/9408234; N. Hata, S. Bludman and P. Langacker, Phys. Rev. D49, 3622 (1994); A. Yu. Smirnov, Ref. [15], p. 105.

[21] B. Pontecorvo, Sov. Phys. JETP 6, 429 (1958); L.M. Krauss and S.L. Glashow, Phys. Lett. 190B, 199 (1987); A. Acker, S. Pakvasa and J. Pantaleone, Phys. Rev. D43, 1754 (1991); V. Barger, R.J.N. Phillips 
and K. Whisnant, Phys. Rev. D43, 1110 (1991); P.I. Krastev and S.T. Petcov, Phys. Rev. Lett. 72, 1960 (1994); Phys. Lett. B299, 99 (1993).

[22] K.S. Hirata et. al., Phys. Lett. B280, 140 (1992).

[23] R. Becker-Szendy et. al., Phys. Rev. D46, 3720 (1992).

[24] For a review of the theoretical status, see T.K. Gaisser, in Ref. 2, p. 309.

[25] Y. Fukuda et. al., Phys. Lett. B335, 237 (1994).

[26] R. Barbieri and A. Dolgov, Phys. Lett. B237, 440 (1990); K. Enqvist, K. Kainulainen and J. Maalampi, Nucl. Phys. B349, 754 (1990); K. Enqvist, J. Maalampi and V. Semikoz, Preprint HU-TFT-95/28 May (1995).

[27] P.J. Lichtfield, in International Europhysics Conference on High Energy Physics, Marsielle, France, (1993).

[28] C. Berger et. al., Phys. Lett. B245, 305 (1990).

[29] R. K. Schaefer and Q. Shafi, Phys. Rev. D, 49, 4990, (1994).

[30] G. Dvali, Q. Shafi, and R.K. Schaefer, 1994, Phys. Rev. Lett., 73, 1886.

[31] C. Athanassopoulos et. al., Preprint nucl-ex/9504003, April (1995).

[32] B. Armbruster et. al., Nucl. Phys. B (Proc. Supple) 38, 235 (1995).

[33] L. Borodovsky et. al., Phys. Rev. Lett. 68, 274 (1992). 
[34] B. Achkar et. al., Nucl. Phys. B 434, 503 (1995).

[35] J. Hill, University of Pennsylvania Preprint, April (1995).

[36] D. Caldwell and R.N. Mohapatra, Phys. Rev. D 48 , 3259 (1993); D50, 3477 (1994); P. Bamert and C.P. Burgess, Phys. Lett. B329, 289 (1994); A. Ioannisian and J.W.F. Valle, Phys. Lett. B322, 93 (1994); A. Joshipura, Z. Phys. C64, 31 (1994); Phys. Rev. D51, 1321 (1995); E.J. Chun, A.S. Joshipura, and A. Yu. Smirnov, hep-ph/9505275; K.S. Babu and S. Pakvasa, Phys. Lett. B172, 360, (1986).

[37] M. de Jong et.al., CERN-PPE/93-131 (1993); N. Armenise et. al., CERN-SPSC/90-42 (1990).

[38] P. Astier et. al., CERN-SPSLC/91-21, CERN-SPSLC/91-48.

[39] B.W. Lee, S. Pakvasa, R.E. Shrock and H. Sugawara, Phys. Rev. Lett. 38, 937 (1977); S.B. Treiman, F. Wilczek and A. Zee, Phys. Rev. D16, 152 (1977); S. Pakvasa, Ref. 2, p. 475.

[40] K.S. Babu, E. Ma and J. Pantaleone, Phys. Lett. B218, 233 (1989).

[41] Particle Data Table, Phys. Rev. D50, 1173 (1994).

[42] K.S. Babu, J.C. Pati and F. Wilczek, hep-ph/9505334, IAS Preprint IASSNS-HEP 95/37.

[43] N.Ushida et. al., Phys. Rev. Lett. 57, 2897 (1986); M. Gruwe et. al., Phys. Lett. B309, 463 (1993). F. Dydak, et al., Phys. Lett., B142, 103, (1984). 
[44] Z. Berezhiani and R.N. Mohapatra, hep-ph/9505385, R. Foot and R.R. Volkas, Preprint UM-P-95/49, RCHEP-95,14, hep-ph/9505359.

[45] E. Ma and P. Roy, Preprint hep-ph/9504342; H. Minakata, hepph/9503417; H. Minakata, hep-ph/9504222; S. Goswami, K. Kar, A. Raychaudhuri, Preprint hep-ph/9505395.

[46] G. Raffelelt and J. Silk, hep-ph/9502306; G. Fuller, J. Primack and Y-Z. Qian, astro-ph/9502081.

[47] J. Gelb and E. Berschinger, Astrophys. J., 436, 491, (1994); C. S. Frenk, S. D. M. White, G. Efstathiou, and M. Davis, Astrophys. J., 351, 10, (1990).

[48] J.R. Bond and A. Szalay, Astrophys. J., 274, 443, (1983).

[49] D. Lynden-Bell, et al., Astrophys. J., 308, 19, (1988)

[50] R. K. Schaefer, in "Frontiers in Physics - From Colliders to Cosmology" Ed. A. Astbury, B. A. Campbell, W. Israel, A. N. Kamal, and F. C. Khanna, World Scientific, Singapore, (1989).

[51] R. K. Schaefer and A. A. de Laix, Bartol Preprint BA-95-25

[52] T. P. Walker, G. Steigman, D.N. Schramm, K.A. Olive, and H.-S. Kang, Astrophys. J., 376, 51, (1991); M.S. Smith, L. H. Kawano, and R.A. Malaney, Astrophys. J. Suppl., 85, 219 (1993).

[53] C. L. Bennett, et al., Astrophys J., 436, 423, (1994). 
[54] S. D. M. White, G. Efstathiou, and C. S. Frenk, Mon. Not. Roy. Ast. Soc., 262, 1023, (1993).

[55] N. Bahcall and R. Cen, Astrophys. J., 408, L77, (1993); N. Bahcall and R. Cen, ApJ, 398, L81, (1992).

[56] A. Biviano, et al., 411, L13, (1993).

[57] A.A. Klypin, talk given at the "Dark Matter Workshop", Univ. Maryland, Oct., 1994

[58] A. A. Klypin and G. Rhee, Astrophys. J., 428, 399, (1994).

[59] B. Fort and J. Miralda-Escude, Astrophys. J., 417, L5, (1993).

[60] L. J. Storrie-Lombardi, R. G. McMahon, M. J. Irwin, C. Hazard, to appear in "ESO Workshop on QSO Absorption Lines", Kluwer, (1995).

[61] K. Lanzetta, et al., Astrophys. J, in press.

[62] G. Kauffman and S. Charlot, Astrophys. J., 430, L97, (1994); H.J. Mo and J. Miralda-Escude, Astrophys. J., 430, L25, (1994).

[63] A. Klypin, S. Borgani, J. Holtzman, and J. Primack, "Damped Lyman Alpha Systems vs. Cold + Hot Dark Matter", Astrophys. J. (in press), (1995).

[64] Note that Ma and Bertschinger (last ref. in [11]) do not entirely agree with this conclusion. The disagreement relates to the different algorithms for identifying dark matter halos in the simulations. 
[65] W. Freedman, et al., Nature, 371, 757, (1994); M. Pierce, D. Welch, R. McClure, S. van den Bergh, R. Racine, P. Stetson, Nature, 371, 385, (1994); A. Saha, et al., Astropys. J., (in press); A. Reiss, W.H. Press, R. Kirshner, Astrophys. J., 438, L17, (1995); M. M. Phillips, Astrophys. J., 413, L105, (1993). J. Maza, M. Hamuy, M. M. Phillips, N. B. Suntzeff, R. Aviles, Astrophys. J., 424, L107, (1994). M. Hamuy, M.M. Phillips, J. Maza, N.B. Suntzeff, R.A. Schommer, R. Aviles, Astron. J., 109, 1, (1995). B. Schmidt, et al., Astrophys. J., 432, 42, (1994).

[66] There are a large number of papers on this subject, and we refer interested readers to general treatments: A. Linde, Particle Physics and Inflationary Cosmology, Harwood Academic, N.Y., 1990; E. Kolb and M. Turner, The Early Universe, Addison-Wesley, New York, (1989).

[67] N. Hata, R. J. Scherrer, G. Steigman, D. Thomas, T.P. Walker, S. Bludman, and P. Langacker, OSU preprint, OSU-TA-6/95

[68] C. Copi, D.N. Schramm, M.S. Turner, 1994, Science (in press).

[69] S.D.M. White, J.F. Navarro, A.E. Evrard, and C.S. Frenk, 1993, Nature, 366, 429 .

[70] A. R. Liddle and D. H. Lyth, Phys. Rept., 231, 1, (1993).

[71] A.R. Liddle, D. H. Lyth, R. K. Schaefer, Q. Shafi, and P.T. Viana, (in preparation). 


\section{$5 \quad$ Figure Captions}

Fig. 1. We show the difference in the rms mass fluctuation amplitude $(\Delta M / M)$ between having the hot dark matter (neutrino) component distributed among 1, 2, or 3 flavors of degenerate neutrinos which correspond to our scenarios ii), iii) and i) respectively, for fitting the neutrino oscillation data. Here $h=0.5, \Omega_{\text {baryon }}=0.05$, and $n=1$, with the spectrum normalized to COBE. The data points are: 1) the mass fluctuation estimate based on cluster formation 54 and 2) the lower limit on the mass fluctuations consistent with the formation of Lyman alpha cloud abundances at high redshift [60]. (The mass at which this lower limit is to be imposed should be somewhere along the constraint line.) Panels a), b), and c) correspond to $\Omega_{\nu}=0.20,0.25$ and 0.30 , respectively. It can be seen that increasing the number of neutrino flavors marginally improves the fit to the cluster data. In panel a) we also show the curve for a pure cold dark matter model.

Fig. 2. The rms mass fluctuation amplitude in a special case of neutrino scenario iii, which has been made consistent with the LSND best fit of $\delta m^{2}=$ $6 \mathrm{eV}^{2}$ in a model with $\Omega_{\nu}=0.25, h=0.5, n=1$. For comparison we also show (from top to bottom) the same $\Omega_{\nu}$ with 1,2 , or 3 degenerate neutrino flavors. The " $2+1$ " and " $1+2$ " model curves are intermediate to the degenerate neutrino cases. The data are the same as in Figure 1.

Fig. 3. The rms mass fluctuation in some selected models with varying

cosmological parameters. We plot curves for two models from scenario iii 
with $\mathcal{N}_{\nu}=2$ for $\left(\Omega_{\nu}=0.20, h=0.5, n=1\right)$ - as advocated in ref. 4 and for $\left(\Omega_{\nu}=0.25, h=0.6, n=0.95\right)$. These two models would predict $\delta m^{2}=5.5$ and $18 \mathrm{eV}^{2}$ at the LSND and KARMEN experiments. The third curve is for a model with $\mathcal{N}_{\nu}=3\left(\Omega_{\nu}=0.25, h=0.6, n=0.98\right)$. Lastly we show a curve from scenario ii) with $\mathcal{N}_{\nu}\left(\Omega_{\nu}=0.20, h=0.5, n=1.00\right)$. Here we have increased $\Omega_{\text {baryon }}$ to reduce the ampitude at cluster scales. As can be seen, it is difficult to determine which neutrino mass scenario is correct based on current cosmological data alone. The data are the same as in Figure 1. 
Figure 1. Mass Fluctuation Amplitude for Degenerate Neutrinos

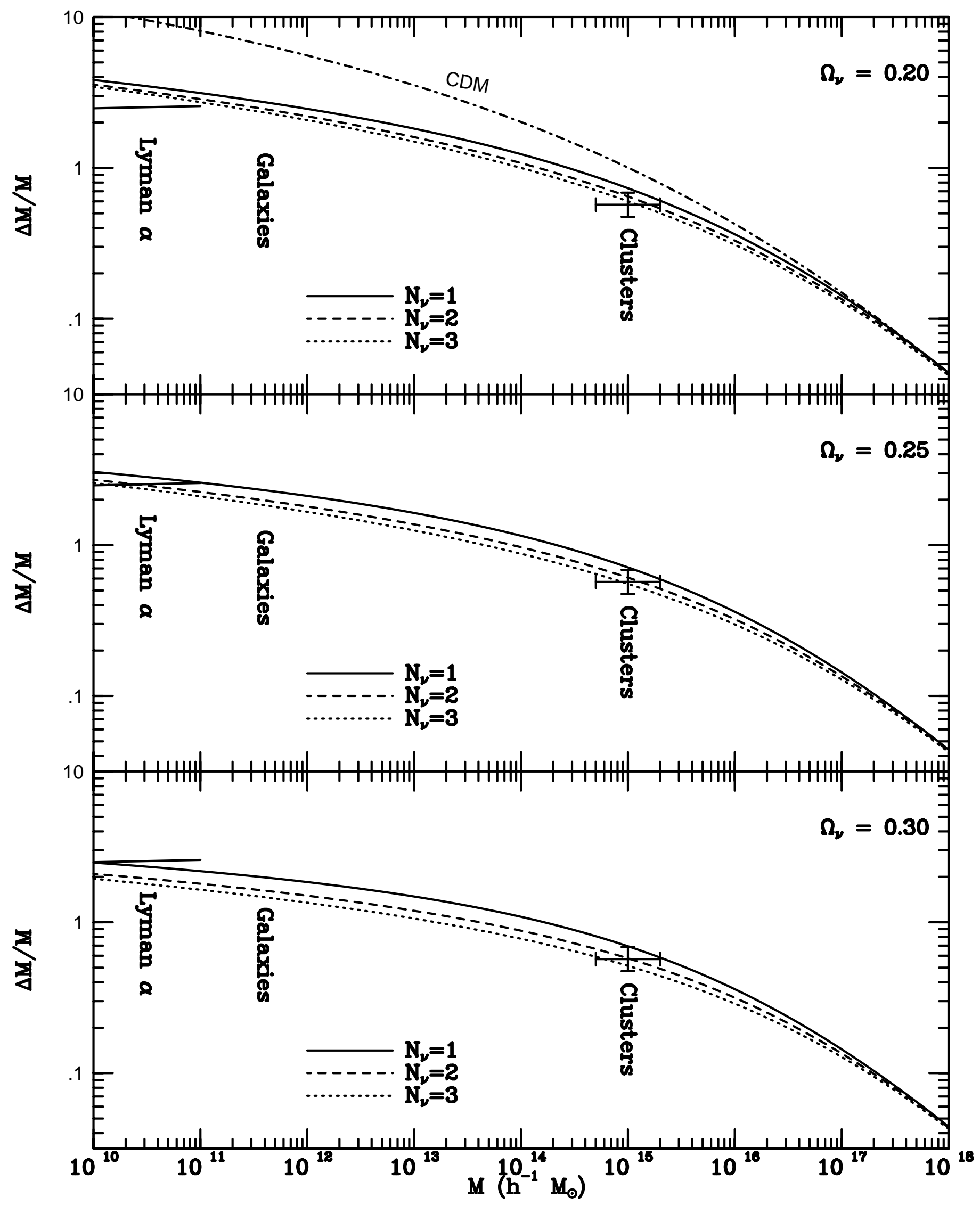

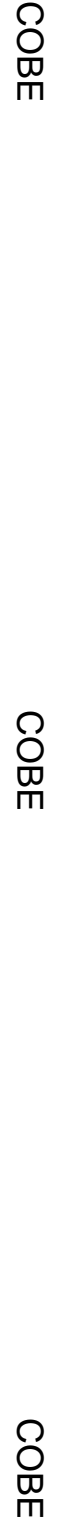


Figure 2. Mass Fluctuation Amplitude

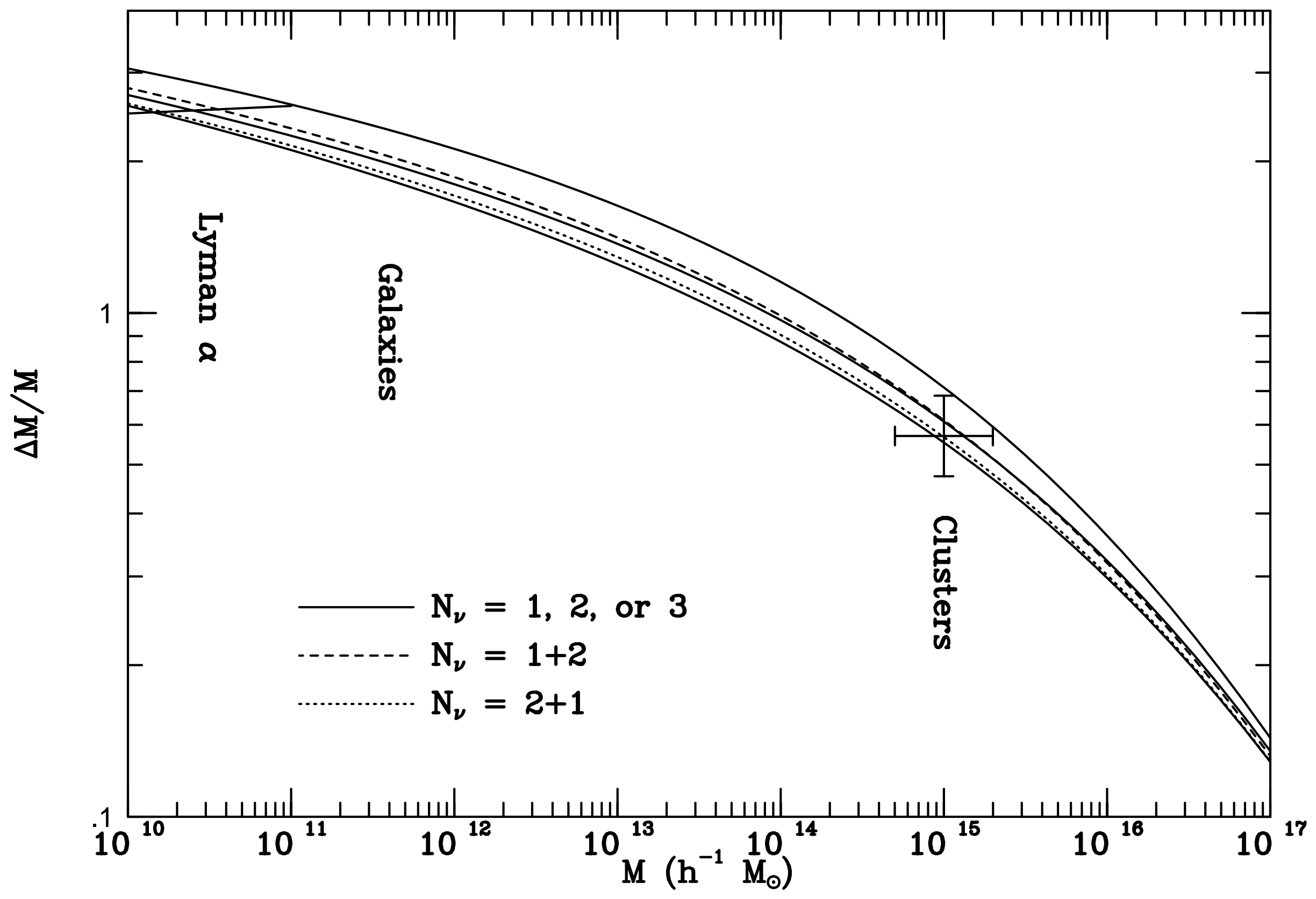


Figure 3. The Effect of Cosmological Parameter Uncertainties

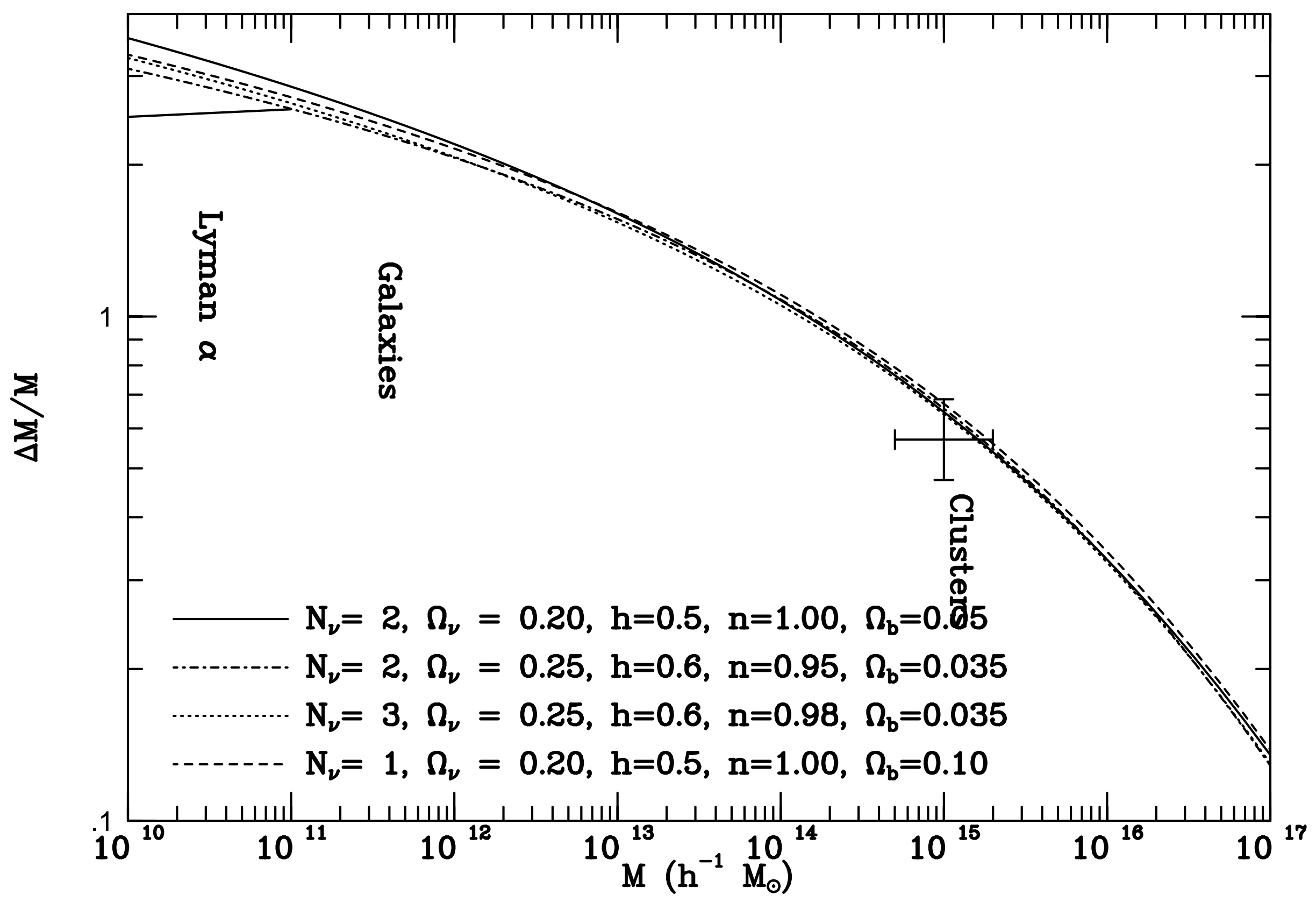

